\title{
Downlink Transmit Power Analysis for Massive MIMO under Imperfect Channel State Information
}

\author{
Lusekelo Kibona, Liu Jian and Liu Yingzhuang \\ Department of Information and Communication Engineering; Huazhong University of Science and Technology, \\ Hubei,Wuhan, China; lusekelo2012@gmail.com, liujian@mail.hust.edu.cn, liuyz@mail.hust.edu.cn
}

\begin{abstract}
Background: The necessity for high-speed data services rises every time in speedy improvement of smart equipment with their uses in wireless network communications, so for the sake of encountering this necessity, massive multiple-inputsmultiple-output has been suggested to increase the spectral efficiency. However, upon increasing spectral efficiency, there is still the Energy Efficiency (E.E) problem. Objectives: In this study, downlink transmit power analysis for massive MultipleInput-Multiple-Output (MIMO) with linear pre-coding schemes under imperfect Channel State Information (CSI) using Time-Division-Duplex (TDD) mode has been carried out to find out which linear pre-coder is energy efficient compared to the other in the presence of estimation channel error which defines the reliability of the channel. Methodology: Derivation of the closed-form expression for transmit power of both pre-coding schemes from achievable sum rates, followed by Monte Carlo simulations and analysis using MATLAB under imperfect CSI have been carried out. Findings: In the presence of channel estimation error $(\zeta)$, our theoretical analysis were found to be in agreement with the simulated results. Furthermore, it was found that, if the ratio of the number of base station antenna $(M)$ to the number of users $(K)$ is large for whatever value of channel estimation error and at constant achievable-sum-rate, the zero-forcing (ZF) precoding scheme gave better results, i.e. uses low amount of transmit power compared to Maximum-Ratio-Transmit (MRT) linear pre-coder and when the ratio $M / K$ is low then MRT gives better results than ZF. Applications/Improvements: The results obtained from this evaluation will lay a foundation or will be used as a framework for suggesting which linear precoding scheme to use according to the number of users $(K)$ required to be served by Base Station (BS) antennas in the presence of $\zeta$.
\end{abstract}

Keywords: Channel Estimation Error, Downlink Transmits Power, Imperfect Channel State Information (CSI), Linear Precoding, Massive Multiple-Input-Multiple-Output (MIMO), Maximum Ratio Transmit (MRT), Time-Division-Duplex (TDD), Zero-Forcing (ZF)

\section{Introduction}

In recent years, substantial technological advancements have arisen and have been used to increase the capacity and performance of wireless network communications. Such improvements are substantial and have carried spectral efficiency of point-to-point communication links close to theoretical limits. Inappropriately, if we wish to encounter the rigorous needs of future generation wireless technology and further than $4 \mathrm{G}$ networks, new or improved features have to be established to combat the anticipated substantial increase in mobile data traffic. To discourse this matter, massive Multiple-Input-Multiple-
Output (MIMO) technology is significant. The main view of massive MIMO is to equip many Base Station (BS) antennas in order to serve numerous users using similar time and frequency resource block. Marzetta ${ }^{1}$ introduced the schemes that solve the problem of non-scalability of standard multiuser MIMO by deploying many BS antennas for a restricted number of users, which also were found to increase system capacity. Massive MIMO is usually operating in the time-division-duplex (TDD) mode, whereby channel reciprocity is utilized to obtain downlink channel estimates from the uplink channel estimates.

*Author for correspondence 
Apart from improving the spectral efficiency and data reliability, massive MIMO also saves the transmit energy, owed to the array gain 2 . The presence of extra base station antennas helps to focus (beam-form) energy/power into even less significant areas of space to get enormous enhancements in capacity and emitted energy efficiency ${ }^{3}$, this capacity also hinges on the propagation surroundings that convey asymptotically orthogonal channels to the terminals $s^{4}$, so generally beam-forming antennas requires little transmission power in comparison with the Omni-directional systems ${ }^{5}$.

Inter-user-interference is problematic for data communication in massive MIMO and thus requiring extra transmit power, so, interference elimination is highly needed at BS ${ }^{6}$. Precoding approaches are important to remove these interferences from BS or user's terminal before transmitting it through the channel ${ }^{7}$. In most cases, linear precoding schemes have received close attention because of their simplicity in implementation and low $\operatorname{cost}^{\stackrel{8}{ }}$. In Massive MIMO, precoding approaches play a crucial part in the signal processing, so many researches have been done in this area, but very few have come up with the impact these precoding schemes plays on the transmit power under the existence of estimation errors. The authors in ${ }^{2}$, used statistical features to relate the performances of MRT and ZF under TDD mode in which they found that, the ZF linear precoding scheme was better than that of MRT, but their analysis did not involve the transmit power. As a result, this paper goes further to analyze the relationship existing between transmit power and channel estimation errors.

For massive MIMO technology, the BS is required to identify the CSI for beam-forming in downlink transmission, and coherent detection in uplink transmission and this channel information must be calculated by users $(K)$ or by BS using Division Duplex (DD). TDD mode is centered explicitly on the channel reciprocity in which the downlink is easily obtained from the uplink channel using reciprocity. Estimation errors in Massive MIMOarevital for the reason that the performance of many wireless communication technologies hinges on the quality of CSI so having the reliable channel without errors ensures the quality transfer of data in the downlink or uplink transmission. Necessarily, it is not expected for any communication to take place without having an error during transmission; this leads to estimation errors in the downlink transmission as shown in Figure $1 \frac{10}{}$.

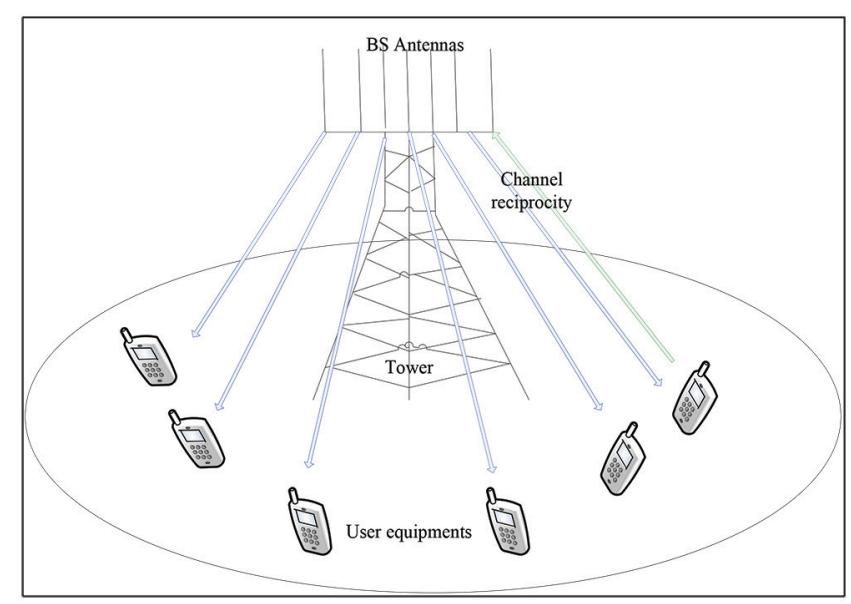

Figure 1. Diagram showing the TDD mode in the downlink transmission.

Most of the works discussed considered either perfect CSI or imperfect CSI in either achievable ergodic rate or spectral efficiency, but none of the works has considered the impact brought by estimation channel errors on the transmit power of massive MIMO.

So the principal aim of this study is to assess the influence of estimation channel errors on the transmit power of massive MIMO under imperfect CSI in the downlink TDD scheme.

This paper offers the following contributions:

- We show the relationship existing between transmit power and estimation error under different linear precoding techniques and then analyze the impact these pre-coders have in the transmit power.

- We compare the impact of estimation channel errors on the precoding schemes in relative to transmit power by changing $M$ antennas and $K$ users.

- We analyze the transmit power theoretically and then compare our results with simulations in the MATLAB for both linear pre-coding systems with different variables.

\section{System Model}

The system model contains $M$ antennas that serve restricted $K$ users in the forward link channel (downlink transmission) under TDD mode (under the assumptions that $K$ users share the frequency-time resources), which assumes channel reciprocity.

The TDD mode is characterized by channel reciprocity whereby reverse channel between the BS and the user 
is a scaled replica of the forward channel whereas, this forward channel is regarded as a $K \times M$ matrix $H$ in which $h_{k}$ acts as a vector between the BS and user $k$ as shown in Figure 2.

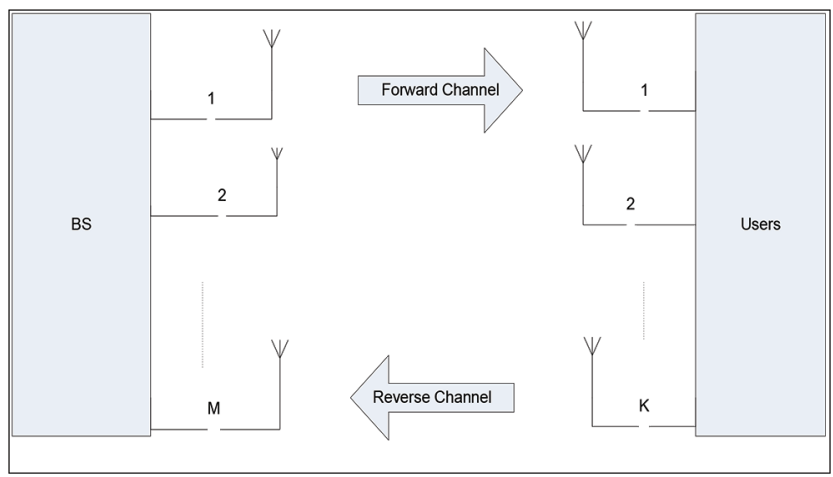

Figure 2. Massive MIMO TDD mode.

Let $F$ be a linear precoding matrix, in which, $F=f_{1}$, $f_{2} \ldots, f_{k}, \ldots, f_{k}$, and $f_{k}$ is the vector pre-coder of user $k$. The signal received vector then is given by:

$$
y=\sqrt{p_{d}} H F x+n
$$

Where $P_{d}$ symbolizes the transmit power in the downlink transmission. $x$ is a $K \times 1$ user vector whereby $x_{k}$ is a symbol for the user $k$. The noise vector $n$ is independent and identically with zero-mean and a unit-variance, i.e., $n \sim C N(0,1)$. The received signal by the user $k$ is:

$$
y k=\sqrt{P_{d}} h_{k} f_{k} x_{k}+\sqrt{P d} \sum_{i=1, i \neq k}^{K} h_{k} f_{i} x_{i}+n
$$

Where $\sqrt{p_{d}} h_{k} f_{k} x_{k}=$ desired signal, $\sqrt{p_{d}} \sum_{i=1, i \neq k}^{K} h_{k} f_{i} x_{i}$ $=$ interference term, and $n=$ noise term

\subsection{Channel estimation}

Estimating channel always do not come with the exact values, so this may lead to estimation errors, which make the channel to be not perfect as in the equation below:

$$
H=\tilde{H}+E
$$

Where the matrix $H \in \mathbb{C}^{K \times M}$ is a MIMO channel with i.i.d complex Gaussian entries. $\tilde{H}$ is an estimated channel matrix attained from Minimum Mean Square Error (MMSE) estimation ${ }^{11}$ also $E=H-\tilde{H}$ is the error matrix in which $H$ and $E$ are always uncorrelated.

Error matrix $E$ elements have the variance of:

$$
\zeta^{2}=M M S E=E\left\{\left[H_{i j}\right]-\left[\tilde{H}_{i j}\right]^{2}\right\}
$$

But for the imperfect CSI, the channel matrix $\tilde{H}$ is given by ${ }^{11}$ :

$$
\tilde{H}=\xi E+\left(\sqrt{1-\xi^{2}}\right) H
$$

Where $\zeta$ is the channel estimation error and $E \sim \mathcal{C N}(0,1)$ denotes the error matrix and is used to find the accuracy of estimation. For $\zeta=0$, means that the channel information is perfect (that is no channel estimation errors) but for $\zeta=1$ means that the channel is entirely imperfect (that is the channel totally contains estimation error).

\subsection{Signal-Interference-Noise Ratio (SINR)}

Is the ratio of signal to the interference plus noise power of the channel, in which for the quality of signal this ratio must be large enough to cancel out any noises and interference associated with the channel? In this description, the SINR for linear precoding techniques under imperfect CSI are derived from ${ }^{12}$,

$$
\operatorname{SINR}_{n}=\frac{S_{n}}{I_{n}+N_{n}}
$$

Where $S_{n}=$ power of the desired signal, $N_{n}=$ noise power and $I_{n}=$ interference. For a received signal $y_{k}$, the SINR of the user $k$ is written as ${ }^{\frac{13}{3}}$ :

$$
\operatorname{SINR}_{k}=\frac{P_{d}\left|h_{k} f_{k}\right|^{2}}{P_{d} \sum_{i=1, i \neq k}^{k}\left|h_{k} f_{i}\right|^{2}+1}
$$

There are two commons (with low complexity) linear precoding techniques usually used in downlink transmission, which are $\mathrm{ZF}$ and MRT/MF respectively.

\subsection{ZF Precoding}

Cancels out inter-user- interference on each user.

$\mathrm{ZF}$ beamforming is given by:

$$
F=\beta\left\{\tilde{H}^{H}\left(\tilde{H} \tilde{H}^{H}\right)^{-1}\right\}
$$

Where $\beta$ is a scaling factor to satisfy the transmit power constraint, and it is given as:

$$
\beta=\sqrt{\frac{1}{\operatorname{tr}\left(A A^{H}\right)}}
$$

Where $A=\tilde{H}^{H}\left(\tilde{H} \tilde{H}^{H}\right)^{-1}$ and $\tilde{H}$ symbolizes the estimated channel matrix.

To satisfy the power control, the precoding matrix must be normalized. 
The approximated kth user $\operatorname{SINR}_{K}^{Z F}$ for Imperfect CSI is given as $\underline{14}, \underline{15}$ :

$$
\operatorname{SINR}_{K}^{Z F}=\frac{\xi^{2} p_{d}(\alpha-1)}{\left(1-\zeta^{2}\right) p_{d}+1}
$$

Where $\alpha=\frac{M}{K}$ then $\operatorname{SINR}_{K}^{Z F}=\frac{\zeta^{2} p_{d}(M-K)}{\left(1-\zeta^{2}\right) K p_{d}+K}$

\subsection{Maximum Ratio Transmission (MRT) Precoding}

Maximizes the signal gain of the planned user $\frac{16}{}$.

The MRT beamforming is given by:

$$
F=\beta\left\{\tilde{H}^{H}\right\}
$$

Where $\beta$ is a scaling element that satisfies the power constraint $E\left\{\operatorname{tr}\left(F F^{H}\right)\right\}=1$ and is given as (6) but $A=\tilde{H}^{H}$.

The approximated kth user $\operatorname{SINR}_{K}^{M R T}$ for large values of $M, K$ and with lower bound vector is given as $\frac{14-15}{15}$ :

$$
\operatorname{SINR}_{K}^{M R T}=\frac{\zeta^{2} p_{d^{\alpha}}}{p_{d}+1}
$$

$$
\text { For } \alpha=\frac{M}{K} \text {, then } \operatorname{SINR}_{K}^{M R T}=\frac{\zeta^{2} p_{d^{\alpha}}}{p_{d}+1}=\frac{\zeta^{2} p_{d} M}{K\left(p_{d}+1\right)}
$$

\subsection{Ergodic Achievable Sum Rate}

Is the average rate in which the transmitter transmits the signals over the channel and it is usually derived from the Shannon formula, by considering the average of the capacity obtained from Shannon theorem from $\frac{12}{}$,

$$
C=\log _{2}(1+\operatorname{SINR})
$$

The above equation can be re-written as:

$$
R_{K}=\log _{2}\left(1+\operatorname{SINR}_{K}\right)
$$

The achievable-sum-rate is given as:

$$
R_{\text {sum }}=\sum_{K=1}^{K} E\left\{R_{K}\right\}
$$

The achievable-sum-rate is approximated as:

$R_{\text {sum }}^{Z F} \approx K \log _{2}\left(1+S I N R_{K}^{Z F}\right)$ Where $P R=$ linear precoder (MRT/ZF)

For ZF pre-coding scheme,

$$
R_{\text {sum }}^{Z F} \approx K \log _{2}\left(1+\operatorname{SINR}_{K}^{Z F}\right)
$$
In which $\operatorname{SINR}_{K}^{Z F}=\frac{\zeta^{2} p_{d}(M-K)}{\left(1-\zeta^{2}\right) K p_{d}+K}$ and substituting
it in Eq. (10).

$$
R_{\text {sum }}^{Z F} \approx K \log _{2}\left(1+\frac{\zeta^{2} p_{d}(M-K)}{\left(1-\zeta^{2}\right) K p_{d}+K}\right)
$$

The achievable-sum-rate for MRT scheme is given as:

$$
R_{\text {sum }}^{M R T} \approx K \log _{2}\left(1+\operatorname{SINR}_{K}^{M R T}\right)
$$

Where $\operatorname{SINR}_{K}^{M R T}=\frac{\zeta^{2} p_{d} \alpha}{p_{d}+1}=\frac{\zeta^{2} p_{d} M}{K\left(p_{d}+1\right)}$ and putting in

$$
R_{\text {sum }}^{M R T} \approx K \log _{2}\left(1+\frac{\xi^{2} p_{d} M}{K\left(p_{d}+1\right)}\right)
$$

\subsection{Transmit Power}

Downlink transmit power is that power released by the base station antenna $(M)$ towards the user equipment antenna $(K)$.

For ZF pre-coding from,

$$
R_{\text {sum }}^{Z F} \approx K \log _{2}\left(1+\frac{\zeta^{2} P_{d}(M-K)}{\left(1-\zeta^{2}\right) K P_{d}+K}\right)
$$

Then the transmit power for $\mathrm{ZF}$ is given as:

$$
P_{d}^{Z F}=\frac{K\left(e^{\frac{R_{s u m}^{Z F}}{K} \ln 2}-1\right)}{\zeta^{2}(M-K)+K\left(1-\zeta^{2}\right)\left(1-e^{\frac{R_{s u m}^{Z F}}{K} \ln 2}\right)}
$$

Proof: See Appendix A.

For MRT precoding:

$$
R_{\text {sum }}^{M R T} \approx K \log _{2}\left(1+\frac{\zeta^{2} P_{d} M}{K\left(P_{d}+1\right)}\right)
$$

Then the transmit power for MRT is given as:

$$
P_{d}^{M R T}=\frac{K\left(e^{\frac{R_{s u m}^{M R T}}{K} \ln 2}-1\right)}{K\left(1-e^{\frac{R_{s u m}^{M N T}}{K} \ln 2}\right)+\zeta^{2} M}
$$

Proof: See Appendix B. 


\section{Theoretical Analysis}

This section provides a theoretical analysis of the expressions derived in the previous section, and by beginning with ZF precoding scheme, consider the following expression for transmit power of ZF,

$$
P_{d}^{Z F}=\frac{K\left(e^{\frac{R_{s u m}^{Z F} \ln 2}{K}}-1\right)}{\zeta^{2}(M-K)+K\left(1-\zeta^{2}\right)\left(1-e^{\frac{R_{s a n}^{Z F}}{K} \ln 2}\right)},
$$

starting with the number of BS, assuming $M$ grows large up to extent that it approaches to infinity, then according to Eq. (16), the transmit power will be tending to zero regardless of the number of users being served which means that, increasing $M$ is an energy efficient way to attain good performance of massive MIMO by using only small amount of transmit power to serve whatever number of users.

$$
\begin{aligned}
& \lim _{M \rightarrow \infty} P_{d}^{Z F}=\lim _{M \rightarrow \infty} \frac{K\left(e^{\frac{R_{s w n}^{Z F} \ln 2}{K}}-1\right)}{\zeta^{2}(M-K)+K\left(1-\zeta^{2}\right)\left(1-e^{\frac{R_{s s m}^{Z F}}{K} \ln 2}\right)} \\
& \approx \lim _{M \rightarrow \infty} \frac{K / M\left(e^{\frac{R_{s u m}^{Z F}}{K} \ln 2}-1\right)}{\zeta^{2}(1-K / M)+K / M\left(1-\zeta^{2}\right)\left(1-e^{\frac{R_{s i m}^{Z F}}{K} \ln 2}\right)} \approx 0
\end{aligned}
$$

As it can be observed in Eq. (16), when $M$ increases, the transmit power tends to decrease, and it reaches zero when $M$ becomes very large indicating that all users are satisfied with even small amount of transmit power. When $M$ is very small compared to the number of users, then the transmit power need to be large to compensate for other users who are not served coherently with the missing transmit antennas and there is a possibility of pilot contamination to occur due to scramble of the signal from the few serving BS antennas, Eq (17) justifies this

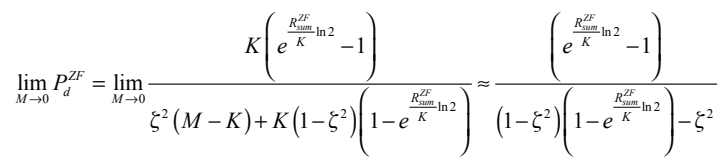

So from Eq. (17), the transmit power will largely depend on the amount of estimation error presents in the channel and the number of users being served, when $M$ is very small compared to $K$.

For the case of estimation error which ranges from 0 to 1 , when it is very close to zero (which means the channel approaches that of perfect CSI), the transmit power needed is very small compared to when the estimation error approaches to 1 (which means the channel is totally imperfect) which yields large transmit power indicating that it is not energy efficient to send signals when the channel is totally imperfect, Eq. (18) presents this issue.

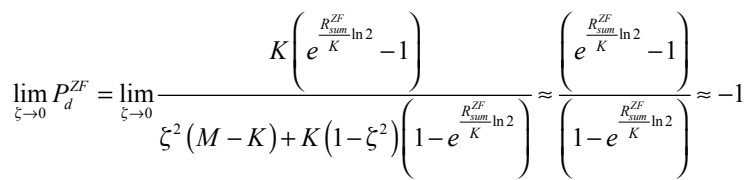

and when $\zeta \rightarrow 1$, the equation is:

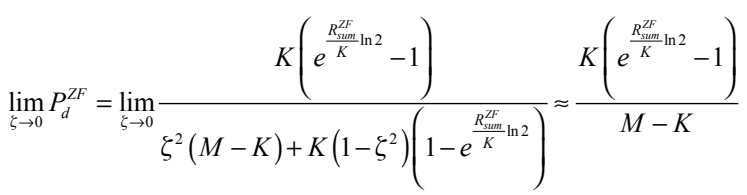

Eq. (19), means that when the value of estimation error grows up to approximately 1, the transmit power also grows depending on the number of BS antennas and the number of users being served by those BS antennas, but in general in the presence of estimation errors, the transmit power used to serve the number of users grows up compared to when the estimation is minimal. Again increasing the number of users brings a negative impact on the transmit power as the power needed to serve those added users will increase as in Eq. (19) and hence cause the degradation in energy efficiency.

The same theoretical analysis for ZF also holds for MRT. Therefore we ignore this part of MRT for space saving.

NB: In comparing ZF and MRT; consider the following equation for $\mathrm{ZF}$,

$$
P_{d}^{Z F}=\frac{K\left(e^{\frac{R_{s i m}^{Z F} \ln 2}{K}}-1\right)}{\zeta^{2}(M-K)+K\left(1-\zeta^{2}\right)\left(1-e^{\frac{R_{s u m}^{Z F} \ln 2}{K}}\right)}
$$

then finding the ratio of $M / K$ we get: 


$$
P_{d}^{Z F}=\frac{\left(e^{\frac{R_{s u m}^{Z F} \ln 2}{K}}-1\right)}{\zeta^{2}(M / K-1)+\left(1-\zeta^{2}\right)\left(1-e^{\frac{R_{s u m}^{Z F} \ln 2}{K}}\right)}
$$

so as $M / K$ becomes very large then:

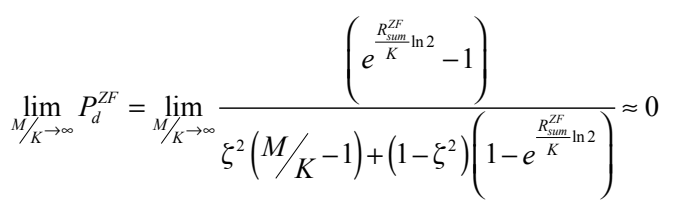

while for MRT,

$$
P_{d}^{M R T}=\frac{K\left(e^{\frac{R_{s u m}^{M R T}}{K} \ln 2}-1\right)}{K\left(1-e^{\frac{R_{s u m}^{M R T}}{K} \ln 2}\right)+\zeta^{2} M} \text { as } M / K
$$

becomes very large then:

$$
\lim _{M / K \rightarrow \infty} P_{d}^{M R T}=\lim _{M / K \rightarrow \infty} \frac{\left(e^{\frac{R_{\text {sum }}^{M R T}}{K} \ln 2}-1\right)}{\left(1-e^{\frac{R_{\text {sum }}^{M R T} \ln 2}{K}}\right)+\xi^{2} M / K} \approx 0
$$

As it can be seen in both equations for ZF and MRT as $M / K$ becomes very large the transmit power tends to be small but in comparison $P_{d}^{Z F}$ is much smaller than that of MRT because it contains many parameters in the denominator. But when $M / K$ is minimal, then from

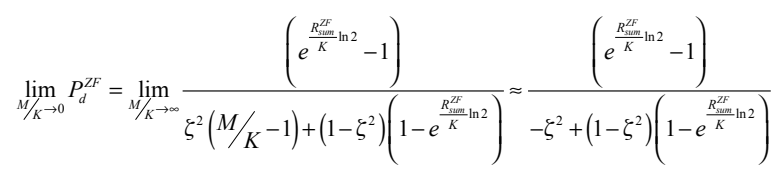

and

$$
\lim _{M / K \rightarrow 0} P_{d}^{M R T}=\lim _{M / K \rightarrow 0} \frac{\left(e^{\frac{R_{\text {sum }}^{M R T}}{K} \ln 2}-1\right)}{\left(1-e^{\frac{R_{\text {sum }}^{M R T}}{K} \ln 2}\right)+\xi^{2} M / K} \approx-1
$$

We found that the transmit power for MRT is very small compared to that of $\mathrm{Zf}$ when the ratio of $M / K$ is minimal, which indicates that, MRT linear precoding scheme performs better when $M / K$ is minimal compared to when $M / K$ is large which favors ZF pre-coder.

\section{Results and Discussions}

In this section, we carry out Monte Carlo simulation to verify the theoretical analysis using MATLAB by varying different parameters to analyze the effect brought by channel estimation errors in the transmit power of Massive MIMO with linear precoding techniques of $\mathrm{ZF}$ and MRT under imperfect CSI.

Figure 3, shows the association between the transmit power and the channel error by which, the transmit power $\left(P_{d}\right)$ was observed to rise when the error raised from 0 to 1 for both MRT and ZF using unchanged conditions, i.e. $R_{z f}=R_{m r t}$ and $K=20$ while $M=128$. Likewise, it was observed that with low values of achievable sum rate $\mathrm{R}=0 \mathrm{bits} / \mathrm{Hz} / \mathrm{s}$, the transmit power was better for both ZF and MRT in comparison with a high amount of achievable sum rate, but ZF linear pre-coder provides better result in comparison with MRT for unchanged conditions.

Increasing the number of serving BS antenna results in a very good results of transmit power in comparison to few antennas for instance, as the ratio of $M / K$ escalates, $P_{d}$ tends to decrease in comparison with low ratio of $M / K$ so as $M$ antennas rises, the transmit power have a tendency to drop gradually, but the relationship with estimation error is not affected as when the estimation error increases similarly the power increases. And once again for increased $M$, e.g., $M=512$, ZF linear precoder was found to outperform MRT precoding scheme, however, for smaller $M$ antennas, the MRT linear pre-coder beat ZF pre-coder as Table 1 shows the extract of Figure 4.

Figure 4 shows the association between $P_{d}$ and $M$ antennas, in which the $P_{d}$ was found to be decreasing as

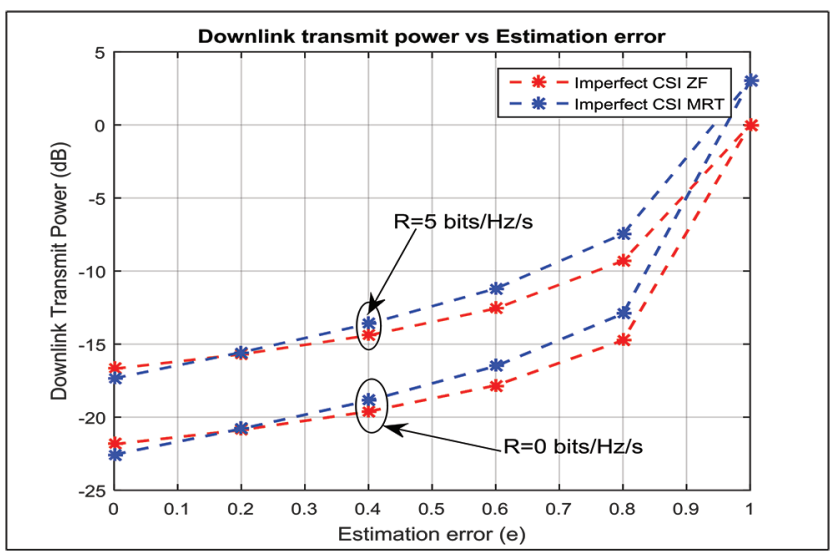

Figure 3. Graph of $P_{d}$ against channel estimation errors $(\zeta)$ when $K=20$ and $M=128$. 
Table 1. Showing the values of downlink transmit power, channel estimation error and $M$ antennas at $R=5 \mathrm{bits} / \mathrm{Hz} / \mathrm{s}$ and $\mathrm{K}=50$

\begin{tabular}{|l|c|c|c|c|c|c|}
\hline \multicolumn{1}{|c|}{$M$} & \multicolumn{2}{|c|}{$M=\mathbf{1 2 8}$} & \multicolumn{2}{c|}{$M=\mathbf{2 5 6}$} & \multicolumn{2}{c|}{$M=\mathbf{5 1 2}$} \\
\hline $\begin{array}{l}\text { Channel } \\
\text { Estimation } \\
\text { error }(e)\end{array}$ & $e=\mathbf{0 . 4}$ & $e=\mathbf{0 . 8}$ & $e=\mathbf{0 . 4}$ & $e=\mathbf{0 . 8}$ & $e=\mathbf{0 . 4}$ & $e=\mathbf{0 . 8}$ \\
\hline$P_{d}^{Z F}(\mathrm{~dB})$ & -13 & -8.5 & -13.5 & -12.5 & -22 & -16 \\
\hline$P_{d}^{M R T}(\mathrm{~dB})$ & -14 & -8 & -12.8 & -11 & -20 & -14 \\
\hline
\end{tabular}

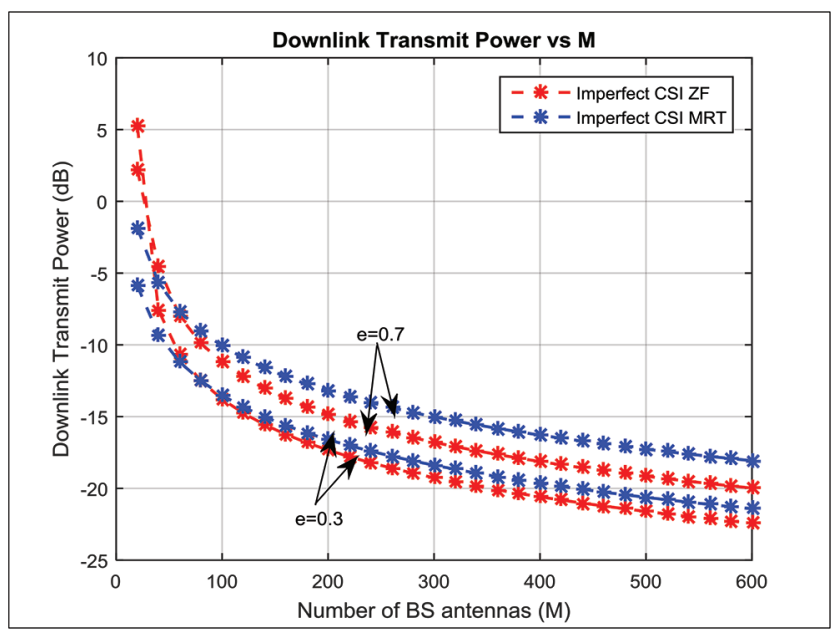

Figure 4. Relationship between $P_{d}$ and $M$ antennas under varying estimation errors.

$M$ grows large which is required for energy efficiency and better performance but again as it can been seen in the Figure 4, with different values of $e$, the $P_{d}$ is best for the channel with small value of channel estimation error in comparison to the one with high value as observed in Figure 4 , the transmit power at $e=0.3$ was better than that with $e=0.7$ having similar condition of $R=5$ bits $/ \mathrm{Hz} / \mathrm{s}$ and $K=20$. However, in both cases, ZF linear pre-coder gave better performance compared to MRT linear precoder.

As it can be observed in the Table 2 increasing $K$ has got unfavorable results on the transmit power subjected to the estimation error and the type of linear pre-coding approach, for the case of MRT, raising $K$ tends to cause $P_{d}^{M R T}$ to drop which is better for energy efficiency in comparison with ZF linear pre-coding in which $P_{d}^{Z F}$ tends to increase as $K$ increases and this commonly occurs when $e$ changes while $M$ and achievable sum rate constant. Therefore, in general, for small $M$ antennas, the MRT outperforms ZF linear pre-coding, and in this case, the concept that MRT performs better, with ratio $M / K$ being low is easily proved.

Table 2. Showing the values of $P_{d}$ with respect to $K$ and with varying estimation error at a constant achievable rate $(R=5$ bits $/ \mathrm{Hz} / \mathrm{s})$ and $M=128$

\begin{tabular}{|l|c|c|c|c|}
\hline \multicolumn{1}{|c|}{$K$} & \multicolumn{2}{|c|}{$K=\mathbf{3 2}$} & \multicolumn{2}{c|}{$K=\mathbf{1 0 0}$} \\
\hline $\begin{array}{l}\text { Channel Estimation } \\
\text { error }(e)\end{array}$ & $e=\mathbf{0 . 3}$ & $e=\mathbf{0 . 7}$ & $e=\mathbf{0 . 3}$ & $e=\mathbf{0 . 7}$ \\
\hline$P_{d}^{Z F}(\mathrm{~dB})$ & -14.6 & -11 & -9 & -5 \\
\hline$P_{d}^{M R T}(\mathrm{~dB})$ & -14.7 & -9.8 & -14.9 & -10 \\
\hline
\end{tabular}

Increasing $M$ had a consequence on $P_{d}$ as observed in the Table 3 that follows and in which $P_{d}$ was observed to be falling as $K$ rises for both linear precoding techniques under the same $M$ and constant achievable-sum-rate. When the estimation error was 0.7 , the ZF precoding scheme performed better even though the number of users was kept increasing compared to MRT but the same case also happens when the estimation error was 0.3 which provided better results for both precoding schemes compared with $e=0.7$, but then again, ZF pre-coder were found to be superior to MRT pre-coder under the same conditions $(R=5$ bits $/ \mathrm{Hz} / \mathrm{s}$ and $M=512)$.

Table 3. Showing the values of $P_{d}$ with respect to $K$ users and with varying $e$ at constant $R=5$ bits $/ \mathrm{Hz} / \mathrm{s}$ and constant $M=512$

\begin{tabular}{|l|c|c|c|c|}
\hline \multicolumn{1}{|c|}{$K$} & \multicolumn{2}{|c|}{$K=32$} & \multicolumn{2}{c|}{$K=\mathbf{1 0 0}$} \\
\hline $\begin{array}{l}\text { Channel Estimation } \\
\text { error }(e)\end{array}$ & $e=\mathbf{0 . 3}$ & $e=\mathbf{0 . 7}$ & $e=\mathbf{0 . 3}$ & $e=\mathbf{0 . 7}$ \\
\hline$P_{d}^{Z F}(\mathrm{~dB})$ & -21.8 & -18 & -21.2 & -17.4 \\
\hline$P_{d}^{M R T}(\mathrm{~dB})$ & -20.9 & -15.9 & -20.9 & -16 \\
\hline
\end{tabular}

\section{Conclusion}

In this article, analysis of the effects of estimation error on the transmit power for massive MIMO under TDD mode has been done in which there are various contrasting results depending on the parameters of analysis and the type of linear precoding scheme used, i.e. ZF and MRT. In theoretical perspectives usually having high amount of transmit power always means poor energy efficiency so we 
expected to have a lower amount of transmit power for a large $M$ that serves $K$ users, but in this article, it happened only in the case that $M$ antennas serving $K$ users were significantly large compared to the $K$ users, and in some cases it was better for ZF precoding scheme than MRT precoding scheme. And in contrast with the results of, our results have proved beyond a reasonable doubt that the presence of estimation errors has significant impact on the energy efficiency for whatever value of the parameter is varied.

For few BS antennas, MRT linear pre-coder was found to be much superior to $\mathrm{ZF}$ at the same situations of estimation error and the achievable-sum-rate $R=5$ bits $/ \mathrm{Hz} / \mathrm{s}$ similar to figure 4 . Therefore, and generally, there is an unlimited relationship between transmit power and the channel estimation errors using linear precoding schemes, as one scheme may be better than the other under certain condition but also the vice versa is true when the conditions changes.

\section{References}

1. Marzetta TL.Noncooperativecellularwirelesswithunlimited numbers of base station antennas, IEEE Transactions on Wireless Communications. 2010; 9(11):3590-600. https://doi.org/10.1109/TWC.2010.092810.091092.

2. Ngo HQ, Larsson EG, Marzetta TL. Energy and spectral efficiency of very large multiuser MIMO systems, IEEE Transactions on Communications. 2013; 61(4):1436-49. https://doi.org/10.1109/TCOMM.2013.020413.110848.

3. Amadori PV. Energy Efficient Large Scale Antenna Systems for 5G Communications and Beyond: UCL, University College London; 2017.

4. Larsson EG, Edfors O, Tufvesson F, Marzetta TL. Massive MIMO for next generation wireless systems, IEEE Communications Magazine. 2014; 52(2):186-95. https://doi.org/10.1109/MCOM.2014.6736761.

5. Chhatani R, Cheeran A. Beamforming and Transmission Power Optimization, Optimization. 2013; 2(1):1-17.

6. Israr A, RaufZ, Muhammad J, Khan F. Performance Analysis of Downlink Linear Precoding in Massive MIMO Systems Under Imperfect CSI, Wireless Personal Communications. 2017; 96(2):2603-19. https://doi.org/10.1007/s11277-0174314-0.
7. Mohan KJ, Gogoi O, Gogoi P. Interference cancellation in massive MIMO base stations with certain precoding techniques in faded environment, Signal Processing and Integrated Networks. 2014; 795-800. https://doi.org/10.1109/SPIN.2014.6777063.

8. Björnson E, Bengtsson $\mathrm{M}$, Ottersten B. Optimal multiuser transmit beam forming: A difficult problem with a simple solution structure, IEEE Signal Processing Magazine. 2014; 31(4):142-48. https://doi.org/10.1109/ MSP.2014.2312183.

9. Raeesi O, Gokceoglu A, Zou Y, Björnson E, Valkama M. Performance analysis of multi-user massive MIMO downlink under channel non-reciprocity and imperfect CSI, IEEE Transactions on Communications. 2018; 66(6):2456-71. https://doi.org/10.1109/ TCOMM.2018.2792017.

10. Tebe PI, Kuang Y, Opare KA, Kponyo JJ. The effect of channel estimation errors on the energy efficiency of downlink massive MIMO systems, Ubiquitous and Future Networks. 2015. https://doi.org/10.1109/ICUFN.2015.7182689.

11. Frigyes I, Bitó J, Bakki P. Advances in mobile and wireless communications: Views of the 16th IST mobile and wireless communication summit, Springer Science and Business Media. 2008.

12. Madhow U. Fundamentals of digital communication: Cambridge University Press; 2008. p. 1-17. https://doi. org/10.1017/CBO9780511807046.

13. Selvan V, Iqbal M, Al-Raweshidy H. Performance analysis of linear precoding schemes for very large Multi-user MIMO downlink system, Innovative Computing Technology. 2014; 219-24. https://doi.org/10.1109/INTECH.2014.6927765.

14. Mi D, Dianati M, Zhang L, Muhaidat S. and Tafazolli R. Massive MIMO Performance With Imperfect Channel Reciprocity and Channel Estimation Error, IEEE Transactions on Communications. 2017; 65(9):3734-49. https://doi.org/10.1109/TCOMM.2017.2676088.

15. Rusek F, Persson D, Lau BK, Larsson EG, Marzetta TL, Edfors O, et al. Scaling up MIMO: Opportunities and challenges with very large arrays, IEEE Signal Processing Magazine. 2013; 30(1):40-60. https://doi.org/10.1109/ MSP.2011.2178495.

16. Pakdeejit E. Linear precoding performance of massive MU-MIMO downlink system, DiVA. 2013, pp. 65. http://urn.kb.se/resolve?urn=urn:nbn:se:liu:diva- 94225 . 


\section{Appendix A:}

From achievable-sum-rate:

$$
R_{\text {sum }}^{Z F} \approx K \log _{2}\left(1+\frac{\zeta^{2} p_{d}(M-K)}{\left(1-\zeta^{2}\right) K p_{d}+K}\right)
$$

converting logarithm in terms of natural logarithm, we get:

$$
R_{\text {sum }}^{Z F} \approx K \frac{\ln \left(1+\frac{\zeta^{2} p_{d}(M-K)}{\left(1-\zeta^{2}\right) K p_{d}+k}\right)}{\ln 2}
$$

then:

$$
\frac{R_{\text {sum }}^{Z F}}{K} \ln 2 \approx \ln \left(1+\frac{\zeta^{2} p_{d}(M-K)}{\left(1-\zeta^{2}\right) K p_{d}+K}\right)
$$

Then:

$$
e^{\frac{R_{s u m}^{Z F}}{K} \ln 2}=1+\frac{\zeta^{2} P_{d}(M-K)}{\left(1-\zeta^{2}\right) K P_{d}+K}
$$

the main aim here is to make transmit power $P_{d}$ as the subject of the formula so:

$$
\begin{aligned}
& \left(\left(1-\zeta^{2}\right) K P_{d}+K\right) e^{\frac{R_{\text {sum }}^{Z F}}{K} \ln 2}=\left(1-\zeta^{2}\right) K P_{d}+K+\zeta^{2} P_{d}(M-K) \\
& \left(1-\zeta^{2}\right) K P_{d} e^{\frac{R_{\text {sum }}^{Z F} \ln 2}{K}}+K e^{\frac{R_{\text {sum }}^{Z F}}{K} \ln 2}=\left(1-\zeta^{2}\right) K P_{d}+K+\zeta^{2} P_{d}(M-K) \\
& K P_{d} e^{\frac{R_{\text {sum }}^{Z F}}{K} \ln 2}-K=\left(1-\zeta^{2}\right) K P_{d}+\zeta^{2} P_{d}(M-K)-\left(1-\zeta^{2}\right) K P_{d} e^{\frac{R_{\text {sum }}^{Z F} \ln 2}{K}}
\end{aligned}
$$

In which $P_{d}=P_{d}^{Z F}$ symbolizes the transmit-power for ZF scheme:

$$
\begin{gathered}
K P_{d}^{Z F} e^{\frac{R_{\text {sum }}^{Z F}}{K} \ln 2}-K=\left(1-\zeta^{2}\right) K P_{d}^{Z F} \\
+\zeta^{2} P_{d}^{Z F}(M-K)-\left(1-\zeta^{2}\right) K P_{d}^{Z F} e^{\frac{R_{\text {sum }}^{Z F}}{K} \ln 2} \\
K\left(e \frac{R_{\text {sum }}^{Z F}}{K} \ln 2-1\right)=P_{d}^{Z F}\left(\zeta^{2}(M-K)+K\left(1-\zeta^{2}\right)\left(1-e \frac{R_{\text {sum }}^{Z F}}{K} \ln 2\right)\right)
\end{gathered}
$$

Then separating $P_{d}^{Z F}$ the equation (14) will be obtained. Appendix B:

From achievable-sum-rate:

$$
R_{\text {sum }}^{M R T} \approx K \log _{2}\left(1+\frac{\zeta^{2} p_{d} M}{K\left(p_{d}+1\right)}\right)
$$

converting logarithm in terms of natural logarithm we get:

$\frac{R_{\text {sum }}^{M R T}}{K} \approx \frac{\ln \left(1+\frac{\zeta^{2} p_{d} M}{K\left(p_{d}+1\right)}\right)}{\ln 2}$ then $\frac{R_{\text {sum }}^{M R T}}{K} \ln 2 \approx \ln \left(1+\frac{\zeta^{2} p_{d} M}{K\left(p_{d}+1\right)}\right)$

by definition of logarithm:

$$
e^{\frac{R_{s u m}^{M R T}}{K} \ln 2}=1+\frac{\zeta^{2} p_{d} M}{K\left(p_{d}+1\right)}
$$

in which:

$$
P_{d}=P_{d}^{M R T}
$$

symbolizes the transmit-power in the downlink transmission for MRT scheme, so:

$$
e^{\frac{R_{s u m}^{M R T}}{K} \ln 2}=1+\frac{\xi^{2} P_{d}^{M R T} M}{K\left(P_{d}^{M R T}+1\right)},
$$

the main aim here is to make transmit power $P_{d}^{M R T}$ as the subject of the formula so:

$$
K\left(P_{d}^{M R T}+1\right) e^{\frac{R_{s u m}^{M R T}}{K} \ln 2}=K\left(P_{d}^{M R T}+1\right)+\zeta^{2} P_{d}^{M R T} M
$$

and then:

$$
K P_{d}^{M R T} e^{\frac{R_{s u m}^{M R T}}{K} \ln 2}+K e^{\frac{R_{s u m}^{M R T}}{K} \ln 2}=K\left(P_{d}^{M R T}+1\right)+\zeta^{2} P_{d}^{M R T} M
$$

collecting like terms together we get:

$$
\begin{aligned}
& K e^{\frac{R_{\text {sum }} \text { MRT }}{K} \ln 2}=K\left(P_{d}^{M R T}+1\right)-K P_{d}^{M R T} e^{\frac{R_{\text {sum }}^{\text {MUn }}}{K} \ln 2}+\zeta^{2} P_{d}^{M R T} M \\
& K\left(e^{\frac{R_{\text {sum }}^{M R T}}{K} \ln 2}-1\right)=P_{d}^{M R T}\left\{K\left(1-e^{\frac{R_{\text {sum }}^{M R T}}{K} \ln 2}\right)+\xi^{2} M\right\}
\end{aligned}
$$

Then making $P_{d}^{M R T}$ the subject of expression in this formula then equation (15) will be obtained. 